\title{
Applications of isotopes in analytical ecogeochemistry
}

\author{
Andreas Zitek ${ }^{1}$ - Johanna Irrgeher ${ }^{2}$ - Thomas Prohaska ${ }^{1}$
}

Received: 2 September 2015 / Accepted: 9 September 2015 /Published online: 7 October 2015

(C) Springer-Verlag Berlin Heidelberg 2015

Analytical ecogeochemistry is an emerging transdisciplinary field of science dealing with the development and application of analytical methods for the advanced and reliable measurement of compounds, elements and isotopes at trace levels in abiotic and biological media. The aim is to answer fundamental questions in different research fields, such as ecology, biology, ecosystem research and environmental science as well as climatology and geology. Evidently, a link to other disciplines can be established as well. Therefore, analytical ecogeochemistry conceptually ties analytical chemistry to the holistic view of the The Total Human Ecosystem according to Naveh [1] (Fig. 1). That is why its research objects also include the human sphere with its technologies and products,

Published in the topical collection Applications of Isotopes in Analytical Ecogeochemistry with guest editors Thomas Prohaska, Andreas Zitek, and Johanna Irrgeher.

Thomas Prohaska

thomas.prohaska@boku.ac.at

Andreas Zitek

andreas.zitek@boku.ac.at

Johanna Irrgeher

johanna.irrgeher@hzg.de

1 VIRIS Laboratory for Analytical Ecogeochemistry, Division of Analytical Chemistry, Department of Chemistry, University of Natural Resources and Life Sciences, Vienna, Konrad-Lorenz-Straße 24, 3430 Tulln, Austria

2 Department of Marine Bioanalytical Chemistry, Institute of Coastal Research, Helmholtz Centre for Materials and Coastal Research, Max-Planck-Straße 1, 21502 Geesthacht, Germany environmental effects created and the environmental services needed.

Whereas ecogeochemistry can be defined as an approach applying ecogeochemical methods to ecological questions [2], analytical ecogeochemistry has an additional and strong focus on the analytical method that is necessary to produce reliable data. This includes the development of new procedures and methods for challenging and emerging research questions as well as the estimation of appropriate uncertainties and a final judgement of the validity of the data produced for the intended use. It usually requires close collaboration between analytical chemists and domain-specific experts in the related scientific disciplines to ensure the sound analytical science and profound domain knowledge needed to solve the problem being investigated.

As a consequence, analytical ecogeochemistry can be described as a real transdisciplinary field of research. Transdisciplinarity itself can be seen as a research strategy bridging the gaps between disciplines and transcending disciplinary boundaries [3]. It becomes active wherever it is impossible to define or attempt to solve problems within the boundaries of subjects or disciplines - or where one goes beyond such definitions [4]. By its nature, transdisciplinary work is characterized by a mutual stimulation and enrichment of the individual disciplines involved leading to changes in their methodological and theoretical perspectives. That is why it has also been called "the sustainable form of interdisciplinarity" [4]. In particular, analytical sciences (or "analytics") have been recognized as driving forces of transdisciplinary research [5].

One of the analytical methods which has gained increasing importance to answer a large variety of different new and challenging questions in different fields of science is the development and application of isotope research. Since the 1960s the so-called traditional light isotopic systems (C, H, 


\section{anthropogenic factors}

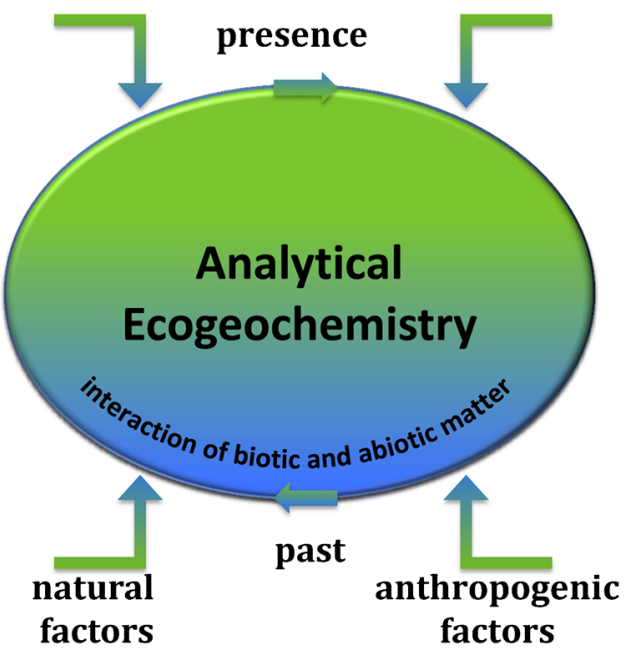

Fig. 1 Visualization of analytical ecogeochemistry as a modern transdisciplinary research field based on the methods of analytical chemistry from the viewpoint of the The Total Human Ecosystem

$\mathrm{O}, \mathrm{N}$ and $\mathrm{S}$ ) have been applied widely (e.g. in ecosystem research), soon followed by the exploration of the so-called traditional heavy isotopic systems $(\mathrm{Sr}, \mathrm{Nd}, \mathrm{Pb}$ and $\mathrm{U})$. With the steady improvement of sensitivity and isotope ratio precision of analytical techniques, the potential of "exotic" isotopic systems has been explored. Isotope ratios, which were historically believed to be invariant in nature, could be proven to show natural variation due to natural mass fractionation. Various examples show both the potential of these systems and the remaining challenges, which need to be tackled to avoid scientific conclusions being drawn from analytical artefacts - underlining the need for sound metrological approaches. In addition to all efforts to study natural processes by the natural variation of the isotopic composition of elements, the use of spike material enriched in a natural isotope has gained importance. The high sensitivity of modern mass spectrometers allows the use of small spike amounts, which can be introduced without interfering with the organism or ecosystem processes, and can be retrieved by new analytical methods such as isotope pattern deconvolution.

We are therefore pleased that we can introduce the conceptual foundations of analytical ecogeochemistry together with examples of transdisciplinary research in this field using isotopes as tools in the topical collection "Applications of Isotopes in Analytical Ecogeochemistry".

Finally, we wish to thank all authors of articles published in this topical collection for submitting their contributions, the referees for their reviews with critical and constructive comments, and the editorial team of Analytical and Bioanalytical Chemistry for their professional collaboration and encouragement to realize this topical collection.

\section{References}

1. Naveh Z (2000) Bioscience 50(4):357-361

2. McMahon KW, Hamady LL, Thorrold SR (2013) Limnol Oceanogr 58(2):697-714

3. Choi BC, Pak AW (2006) Clin Invest Med 29(6):351-364

4. Mittelstraß J (2011) J Humanit Soc Sci 15(4):329-338

5. Koizumi H (2001) Neuroendocrinol Lett 22:219-221

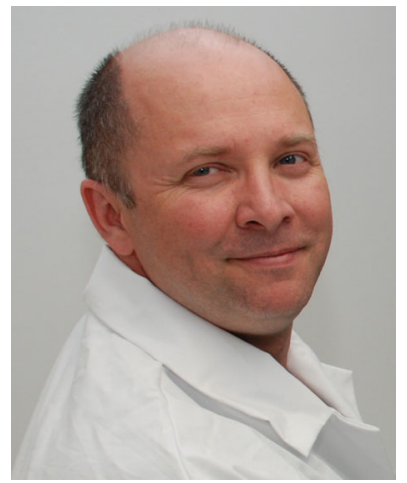

Andreas Zitek has been a postdoctoral research scientist at the University of Natural Resources and Life Sciences, Vienna, since 2009 and has the role of a key researcher in the VIRIS Laboratory for Analytical Ecogeochemistry in the Department of Chemistry, Division of Analytical Chemistry. As a landscape ecologist, he engages in the definition and development of the transdisciplinary research area of analytical ecogeochemistry and closely collaborates with analytical chemists in this field. His current major research interest is the application of elemental and isotopic analyses to basic questions in landscape ecology, mainly in the field of aquatic ecology (aquatic ecogeochemistry), with special focus on the chemical analysis of hard parts of freshwater fish. Another part of his work deals with chemical imaging from the micrometre (small compartment) to kilometre (landscape, isoscape) scale by the application of geographic information system methods and hyperspectral imaging in the near-infrared. He recently co-edited the book Sector Field Mass Spectrometry for Elemental and Isotopic Analysis together with Thomas Prohaska, Johanna Irrgeher and Norbert Jakubowski. 


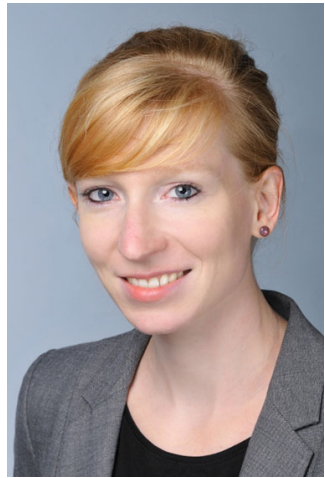

Johanna Irrgeher is currently a postdoctoral researcher in the field of analytical chemistry at the Helmholtz Centre for Materials and Coastal Research in Geesthacht. She obtained her PhD degree (awarded by the Austrian Academy of Sciences) for her work on stable strontium isotope ratio analysis by laser ablation multiple collector inductively coupled plasma mass spectrometry in the VIRIS Laboratory for Analytical Ecogeochemistry at the University of Natural Resources and Life Sciences, Vienna, in 2013, and was a postdoctoral researcher there until 2015. She was a visiting researcher at the Institute of Isotopes in Budapest (Hungary), the National Cheng Kung University in Tainan (Taiwan) and the National Research Council Canada in Ottawa (Canada). Since 2014 Johanna has been an associate member of the IUPAC Commission on Isotope Abundances and Atomic Weights as well as Chair of the IUPAC Subcommittee on Isotope Abundance Measurements. Her current research focuses on analytical method development for elemental and isotopic analysis in the field of analytical ecogeochemistry dealing with aquatic and terrestrial ecosystems.

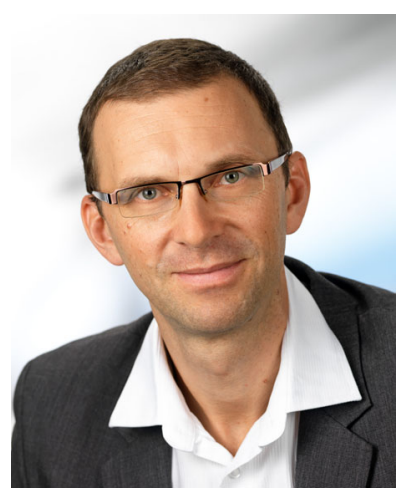

Thomas Prohaska is Professor of Analytical Chemistry at the University of Natural Resources and Life Sciences, Vienna. His research focuses on elemental and isotopic mass spectrometry and covers food provenance and quality, environmental biomonitoring, migration studies and medical and forensic sciences. He is the author of more than 100 peer-reviewed scientific publications. He is a member of the board of directors of the Austrian Society for Chemistry and Physics, Secretary of the IUPAC Commission on Isotopic Abundances and Atomic Weights, a member of the Austrian Academy of Sciences, and a member of the EURACHEM Education and Training Working Group. 